\title{
Calcium Sensing by Recoverin : Effect of Protein Conformation on Ion Affinity
}

\section{Timr, Stepan}

2018-04-05

Timr , S , Kadlec , J , Srb , P , Ollila , O H S \& Jungwirth , P 2018 , ' Calcium Sensing by Recoverin : Effect of Protein Conformation on Ion Affinity ' , Journal of Physical Chemistry Letters , vol. 9 , no. 7 , pp. 1613-1619 . https://doi.org/10.1021/acs.jpclett.8b00495

http://hdl.handle.net/10138/324026

https://doi.org/10.1021/acs.jpclett.8b00495

acceptedVersion

Downloaded from Helda, University of Helsinki institutional repository.

This is an electronic reprint of the original article.

This reprint may differ from the original in pagination and typographic detail.

Please cite the original version. 


\title{
Calcium Sensing by Recoverin: Effect of Protein Conformation on Ion Affinity
}

Štěpán Timr ${ }^{\dagger, 1}$, Jan Kadlec ${ }^{\dagger}$, Pavel Srb ${ }^{\dagger}$, O. H. Samuli Ollila ${ }^{\dagger,}$, and Pavel Jungwirth ${ }^{\dagger *}$

${ }^{\dagger}$ Institute of Organic Chemistry and Biochemistry, Czech Academy of Sciences, Flemingovo nám. 2,

16610 Prague 6, Czech Republic

${ }^{\sharp}$ Institute of Biotechnology, University of Helsinki, Helsinki, Finland

"email: pavel.jungwirth@uochb.cas.cz

Keywords: Recoverin, EF hand, myristoyl switch, molecular dynamics simulations, calcium signaling.

\section{TOC Graphic:}

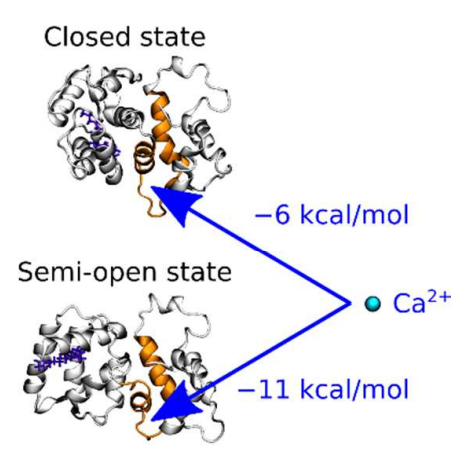

\begin{abstract}
:
The detailed functional mechanism of recoverin, which acts as a myristoyl switch at the rod outer-segment disk membrane, is elucidated by direct and replica-exchange molecular dynamics. In accord with NMR structural evidence and calcium binding essays, simulations point to the key role of enhanced calcium binding to the EF3 loop of the semi-open state of recoverin as compared to the closed state. This two- to four-order decrease in calcium dissociation constant stabilizes the semi-open state in response to the increase of cytosolic calcium concentration in the vicinity of recoverin. A second calcium ion then binds to the EF2 loop and, consequently, the structure of the protein changes from the semi-open to the open state. The latter has the myristoyl chain extruded to the cytosol, ready to act as a membrane anchor of recoverin.
\end{abstract}

\footnotetext{
${ }^{1}$ Present address: Laboratoire de Biochimie Théorique, CNRS UPR9080, Institut de Biologie PhysicoChimique, Université Paris Diderot, Sorbonne Paris Cité, 13 rue Pierre et Marie Curie, 75005 Paris, France
} 
Numerous physiological processes in living cells are regulated by changes in the cytoplasmic concentration of calcium ions. ${ }^{1} \mathrm{Ca}^{2+}$ is sensed by specialized calcium-binding proteins, such as calmodulin, synaptotagmin, various members of the broad protein family of neuronal calcium sensor (NCS), and others. ${ }^{2-3}$ The fact that the concentration of a single ionic species can regulate diverse metabolic and signal transduction pathways has been linked to distinct calcium affinities of the different calcium sensors, which respond to distinct ranges of $\mathrm{Ca}^{2+}$ concentrations. ${ }^{3}$ A full understanding of this diversity requires atomistic insights into the mechanism of calcium detection and the associated conformational transitions of the individual calcium-sensing proteins.

In the present work, we focus on recoverin, which is a globular $23 \mathrm{kDa}$ NCS protein expressed in photoreceptor cells of the vertebrate retina. Recoverin is involved in a calcium-dependent feedback loop modulating the amount of response to a visual stimulus. ${ }^{4}$ Upon an increase in the intracellular $\mathrm{Ca}^{2+}$ concentration, a calcium-induced myristoyl switch allows the protein to associate reversibly with the rod outer-segment (ROS) disk membrane and to inhibit there the rhodopsin kinase..$^{3-5}$ This prolongs the lifetime of the activated state of rhodopsin, which is the light-sensitive receptor protein expressed in high amounts in ROS membranes. ${ }^{5}$

The structure of recoverin consists of two domains connected via a flexible linker (Fig. 1). Both domains contain two EF hands, which are evolutionarily conserved helix-loop-helix motifs shared with the other members of the NCS family and other calcium-binding proteins. ${ }^{6}$ In recoverin, the second and the third EF hands can bind calcium, whereas calcium binding to the remaining two EF hands is blocked due to inactivating substitutions in their loops. At low intracellular $\mathrm{Ca}^{2+}$ concentrations, the two binding sites for calcium are rarely occupied and, consequently, the myristoyl group of recoverin is sequestered inside the $\mathrm{N}$-terminal domain of the protein in a cavity formed by five $\alpha$-helices (Fig. 1A). ${ }^{7}$ When the cytoplasmic $\mathrm{Ca}^{2+}$ concentration increases, binding of two $\mathrm{Ca}^{2+}$ ions results in a structural rearrangement of the two domains and in the exposure of the myristoyl group to the cytoplasm (Fig. 1C). ${ }^{8-9}$ Moreover, the conformational transition connected with myristoyl extrusion from the hydrophobic core of the protein creates a binding pocket for rhodopsin kinase in the N-terminal domain., 5 
While both the initial conformation without calcium and the final conformation binding two $\mathrm{Ca}^{2+}$ ions have been determined by solution $\mathrm{NMR}^{7,9}$ (see Figs. 1A,C) and while the atomistic details of the membrane association process have been elucidated by solid-state $\mathrm{NMR}^{10}$ and molecular simulations, ${ }^{11}$ the interplay between calcium binding and changes in recoverin conformation remains to be clarified. Previous models derived from calcium-binding assays suggested that the calcium affinities of the EF hands in the closed myristoyl-sequestering state (denoted as T) are dramatically weaker than those in the open myristoyl-exposing state (labelled as R). ${ }^{12}$ In fact, while the calcium dissociation constants in the R state were found to be equal to 0.11 and $6.9 \mu \mathrm{M}$ for the EF2 and EF3 loops, respectively, these dissociation constants were estimated to be about three to four orders of magnitude higher in the $\mathrm{T}$ state. ${ }^{12}$

Recent measurements of the ${ }^{15} \mathrm{~N}$ NMR relaxation dispersion ${ }^{13}$ revealed that recoverin undergoes a millisecond-timescale conformational dynamics even in the absence of $\mathrm{Ca}^{2+}$ in the solution. The relaxation dispersion measurements pointed to the existence of a minor population of structures $(>1 \%)$ distinct from the $\mathrm{T}$ state but coexisting with it even in $\mathrm{Ca}^{2+}$-free solution. The signal from this minor species became stronger when a trace amount of $\mathrm{Ca}^{2+}$ was added, indicating that the species represents an intermediate state (I) along the myristoyl-switch pathway. This led the authors to the formulation of a three-state model involving T, I, and R states. ${ }^{13}$ Considering also the results of previous NMR experiments and $\mathrm{Ca}^{2+}$-binding assays, it was concluded that the most likely transition pathway starts with a conformational transition from $\mathrm{T}$ to $\mathrm{I}$, which is followed by the binding of two $\mathrm{Ca}^{2+}$ ions to I. ${ }^{13}$ Finally, after these two $\mathrm{Ca}^{2+}$-binding events, recoverin loaded with two $\mathrm{Ca}^{2+}$ ions evolves from I to R. According to this model, the I state, despite being short-lived, forms a crucial element of the transition pathway and the knowledge of its structure is thus critical for understanding the mechanism of the $\mathrm{Ca}^{2+}$-induced myristoyl switch.

The exact structure of the I state could not be determined from the NMR measurements owing to its short-lived nature. Nevertheless, the positioning of residues undergoing chemical exchange ${ }^{13}$ strongly suggests that the $\mathrm{T} \rightarrow \mathrm{I}$ transition involves rotation of the two protein domains. Nonetheless, the myristoyl group in the I state still appears to be hidden inside the N-terminal domain. ${ }^{13}$ The experimental data ${ }^{13}$ thus indicate that the structure of the I state is similar to that of the calcium-loaded 
E85Q recoverin mutant (PDB ID 1LA3), ${ }^{14}$ which has a mutation in the EF2 loop (glutamate 85 replaced with a glutamine), preventing it from binding the second calcium ion. As a consequence, the NMR structure of this mutant obtained at a high concentration of $\mathrm{Ca}^{2+}$ only contains a single $\mathrm{Ca}^{2+}$ ion, which is bound to EF3. The two domains of this mutant structure (see Fig. 1B) are rotated similarly to those of the fully $\mathrm{Ca}^{2+}$-loaded structure (Fig. 1C); however, the myristoyl moiety is still hidden inside the $\mathrm{N}$-terminal domain, as in the $\mathrm{Ca}^{2+}$-free recoverin.

In this study, we employ direct and enhanced-sampling molecular dynamics (MD) simulations to explore the conformation and the dynamical properties of the intermediate I state, assuming its structural similarity to the semi-open E85Q mutant, as argued above. Moving beyond the traditional assumption of allosteric models, namely that calcium binding should not significantly perturb the structure of the given state, we examine the differences in the structure of the I state with no $\mathrm{Ca}^{2+}$ bound vs. a state where $\mathrm{Ca}^{2+}$ is present in the EF hand 3 of the protein. Moreover, by performing replica-exchange umbrella sampling (REUS) ${ }^{15}$ simulations, we quantify the dependence of the $\mathrm{Ca}^{2+}$ binding affinity on protein conformation. In particular, we inspect the validity of the assumption that the $\mathrm{Ca}^{2+}$ affinity to the EF3 loop increases considerably upon a conformational transition from the closed state to the semi-open one.

In order to investigate the conformational dynamics of the probable intermediate state binding $\mathrm{a} \mathrm{Ca}^{2+}$ ion, we performed first a $1 \mu$ s direct MD simulation, started from a back-mutated and relaxed structure of the calcium-loaded recoverin mutant (PDB ID 1LA3) ${ }^{14}$. We compared the resulting trajectory with that obtained for the $\mathrm{Ca}^{2+}$-free closed state (PDB ID 1IKU) ${ }^{7}$. Neither of the two trajectories revealed significant changes in the overall protein structure. For both states, the root-meansquare deviation (RMSD) of the C $\alpha$ backbone atoms from the initial structure stabilized around 0.4 $\mathrm{nm}$ (Fig. S1). In both trajectories, recoverin retained its overall conformation, as was also documented by a generally good agreement between calculated and experimental chemical shifts (see Figs. S2 and $\mathrm{S} 3$ in SI). Moreover, ${ }^{15} \mathrm{~N}$ spin relaxation rates computed from the two trajectories compared favorably with experimental data ${ }^{13}$ (Figs. S4 and S5 in SI). The good agreement with experimental data indicates that the dynamics of the system monitored through chemical shifts (reporting on timescales up to 
milliseconds) and ${ }^{15} \mathrm{~N}$ spin relaxation rates (ps-ns timescale) was well described by the MD trajectories.

In both simulations, the N-terminal domain, sequestering the myristoyl group, appeared less stable than the C-terminal domain. In particular, in the semi-open state, the N-terminal helix A became shorter by about eight residues in the course of the trajectory as compared to the initial structure and also in comparison to the closed state (see Fig. S6). This result suggests that the domain rotation lowers the stability of the N-terminus, facilitating the extrusion of the myristoyl moiety, as predicted in Ref. ${ }^{14}$. In addition, within tens of nanoseconds after the start of the simulation of the semi-open state, the flexible interdomain linker changed its conformation becoming more S-shaped. Similarly, the helix D of EF2 in the closed-state structure became temporarily destabilized during the initial 100 ns, and it also moved farther away from the C-terminal domain. Such a motion of this helix and potentially also its partial unfolding may accompany the rotation of the two domains and allow this helix to avoid clashing with the helix G of EF3.

To test the effect of $\mathrm{Ca}^{2+}$ on the conformation of the semi-open state, we repeated the corresponding simulation after removing the $\mathrm{Ca}^{2+}$ ion from EF3. We found that the removal of the calcium cation resulted in restructuring of the EF3 loop, which translated into a conformational change of the whole EF hand and, ultimately, into a substantial relative rotation of the two protein domains (see Figs. S7 and S8). The domain rotation was mediated by the interaction of the EF2 and EF3 loops, with the now more expanded EF3 loop pushing against the neighboring EF2 loop. Taken together, this MD trajectory indicates that the conformation of the EF3 loop, altered by the presence or absence of a $\mathrm{Ca}^{2+}$ ion, has a considerable effect on the overall structure of the intermediate state.

Since at the time scale of the direct simulation the system never returned to the original geometry, we further explored the allosteric effect of the EF3 loop on the structure of recoverin in the semi-open state by performing a replica exchange with solute scaling (REST2) simulation ${ }^{16}$ of the same $\mathrm{Ca}^{2+}$-free semi-open structure. In this simulation, we selectively heated up the atoms of the empty EF3 loop to enhance the sampling of its different possible conformations appearing without the stabilizing presence of $\mathrm{Ca}^{2+}$. We confirmed that the absence of $\mathrm{Ca}^{2+}$ from EF3 led to an expansion of the structure of the EF3 loop, as measured by its radius of gyration, which increased from $0.65 \mathrm{~nm}$ 
(RMSD of $0.02 \mathrm{~nm}$ ) to $0.76 \mathrm{~nm}$ (RMSD of $0.06 \mathrm{~nm}$ ). Thus, the average radius of gyration shifted toward a value typical of the $\mathrm{Ca}^{2+}$-free closed state of the protein of $0.83 \mathrm{~nm}$ (RMSD of $0.04 \mathrm{~nm}$ ). The EF3 loop became more disordered, with the majority of conformations approaching those found in the closed state (see Fig. S10). In overall, the semi-open state of recoverin without $\mathrm{Ca}^{2+}$ displayed a significantly increased conformational variation as compared to the state with $\mathrm{Ca}^{2+}$. This effect was particularly pronounced in the region of the EF hands 3 and 2, as well as in the inter-domain linker (see Figs. S11 and S12).

The increased structural variation of the semi-open state lacking $\mathrm{Ca}^{2+}$ was also reflected in a broader distribution of the mutual orientations of the two protein domains (Fig. S13). While certain structures still resembled those sampled with $\mathrm{Ca}^{2+}$ bound in EF3, many others featured markedly different conformations of the EF3 hand and, in turn, different orientations of the two domains (see Fig. 1E). This also affected the position and conformation of the EF2 loop, which was shifted by interactions with the disordered neighboring EF3 loop. In contrast, an analogous REST2 simulation performed for the $\mathrm{Ca}^{2+}$-free closed state confirmed a considerably higher overall stability of this structure compared to the calcium-free semiopened state (see Fig. S14). The same was true for the conformations of the EF hands 2 and 3 (Fig. S11, bottom), although a more detailed inspection revealed fluctuations in the relative angle between the helices D and E of EF2, inducing moderate changes in the relative orientation of the two protein domains in the closed state. 


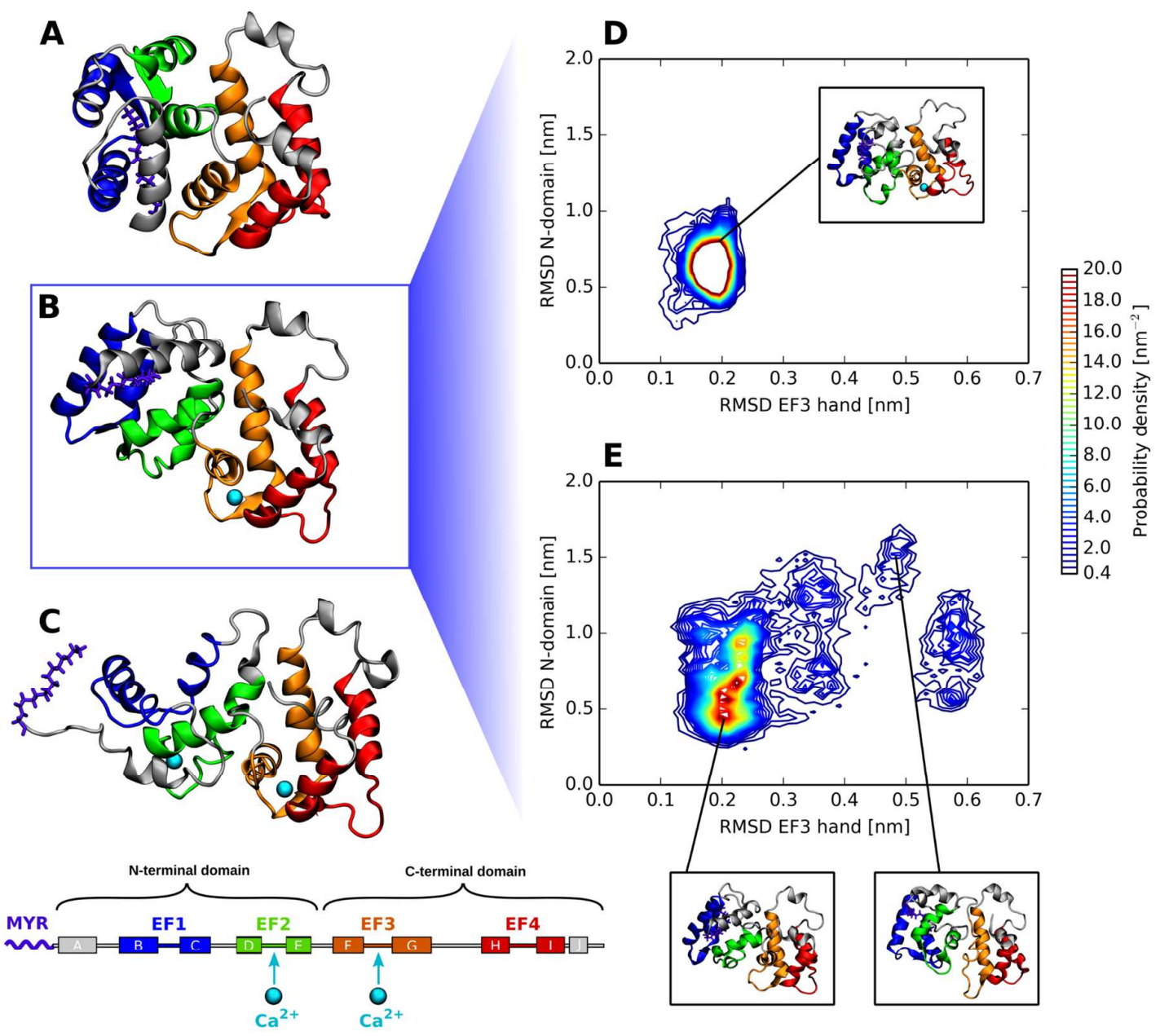

Figure 1. (A) NMR structure of the closed state of recoverin with no calcium bound (geometry no. 1 of PDB ID 1IKU). ${ }^{7}$ (B) NMR structure of a semi-state of recoverin containing a single $\mathrm{Ca}^{2+}$ ion (E85Q recoverin mutant, geometry no. 1 of PDB ID 1LA3). ${ }^{14}$ (C) NMR structure of the open state with two calcium ions bound (geometry no. 1 of PDB ID 1JSA). ${ }^{9}$ The $\alpha$-helices of recoverin are labelled alphabetically according to their order in the protein sequence. (D-E) 2D histograms showing the correlation between the conformation of the EF hand 3, as measured by the Ca RMSD of residues 99-132 from the initial structure, and the relative orientation of the two protein domains, quantified by the C $\alpha$ RMSD of the N-terminal domain (residues 2-91) after an alignment of the C-terminal domain (residues 122-189) to the initial structure. A comparison of results from a $1 \mu$ sirect MD trajectory of the semi-open state with $\mathrm{Ca}^{2+}(\mathrm{D})$ and a REST2 simulation of the semi-open state without $\mathrm{Ca}^{2+}(\mathrm{E})$. 
Without $\mathrm{Ca}^{2+}$ in EF3, the semi-open state of recoverin samples a broader range of EF3 hand conformations and domain orientations.

Previous experiments ${ }^{12}$ revealed that in the open state of recoverin the $\mathrm{Ca}^{2+}$ affinity of $\mathrm{EF}$ hand 3 is higher than that of EF2. On the basis of this observation, it was hypothesized that upon an increase in the intracellular $\mathrm{Ca}^{2+}$ concentration calcium binds first to $\mathrm{EF} 3 .{ }^{12,14}$ As demonstrated by the NMR structure of the semi-open mutant (PDB ID 1LA3), ${ }^{14}$ such calcium binding promotes the largescale domain rotation associated with the myristoyl switch. ${ }^{14}$ However, it remains unclear whether $\mathrm{Ca}^{2+}$ primarily binds to the closed state, triggering the domain rotation, or whether its role is rather to stabilize the semi-open state. While the $\mathrm{Ca}^{2+}$ affinity of the closed state was roughly estimated from fits of $\mathrm{Ca}^{2+}$ binding curves to be about $10^{3}-10^{4}$ times lower than that of the open state (the latter corresponding to a free energy difference of $4.1-5.5 \mathrm{kcal} / \mathrm{mol}),{ }^{12}$ it was never measured directly.

To examine how the $\mathrm{Ca}^{2+}$ affinity of the EF3 loop is altered by the structural transition from the closed state to the semi-open one, we determined computationally the standard $\mathrm{Ca}^{2+}$ binding free energies $\Delta G_{\mathrm{b}}^{\mathrm{o}}$ for a set of recoverin structures representing both states. This was done by performing replica exchange umbrella sampling (REUS) simulations ${ }^{15}$ along a coordinate describing the displacement of the $\mathrm{Ca}^{2+}$ ion from the center of the EF loop and by applying a standard volume correction (see SI for more details). For those structures of recoverin that contained initially no $\mathrm{Ca}^{2+}$, we investigated the $\Delta G_{\mathrm{b}}^{\mathrm{o}}$ of $\mathrm{Ca}^{2+}$-protein complexes formed spontaneously during a 50 ns direct MD simulation after bringing a $\mathrm{Ca}^{2+}$ ion to the proximity of EF3. The restructuring of EF3 to optimally accommodate the $\mathrm{Ca}^{2+}$ ion may entail crossing substantial barriers in potential energy; therefore, the $\mathrm{Ca}^{2+}$-recoverin complex formed at a $50 \mathrm{~ns}$ time scale may not correspond to the lowest-energy state. For this reason, we investigated additional complexes for which the coordination number of $\mathrm{Ca}^{2+}$, calculated in the following as the number of protein oxygen atoms within $0.3 \mathrm{~nm}$ from the center of the $\mathrm{Ca}^{2+}$ ion, was increased to 6 , i.e., to a value observed in the NMR structures of calcium-loaded recoverin. ${ }^{9,} 14$ This was accomplished by running a biased MD simulation before the actual REUS simulation (see SI for more details). 
For the closed state of recoverin, we found the $\mathrm{Ca}^{2+}$ binding to be relatively weak, with $\Delta G_{\mathrm{b}}^{\mathrm{o}}$ of $-4.8 \mathrm{kcal} / \mathrm{mol}$ for a spontaneously formed complex. This value changed to $-6.3 \mathrm{kcal} / \mathrm{mol}$ after increasing the coordination number of calcium in a biased simulation (Table 1). In the former case, the $\mathrm{Ca}^{2+}$ cation was bound to three- to four oxygen atoms of the loop residues in the free-energy minimum (see Fig. 2), which gives a significantly smaller coordination values than 6 found in the NMR structures of the semi-open and open states. ${ }^{9}{ }^{14}$ In the latter case, we observed an average $\mathrm{Ca}^{2+}$ coordination number of 5 for the free-energy minimum and the EF loop 3 partially reverted to the elongated conformation typical for the $\mathrm{Ca}^{2+}$-free structure of the closed state.

For the $\mathrm{Ca}^{2+}$-loaded semi-open state, the $\mathrm{Ca}^{2+}$ binding was significantly stronger than for the closed state. The value of $\Delta G_{\mathrm{b}}^{\mathrm{o}}$ of $-11.1 \mathrm{kcal} / \mathrm{mol}$ obtained for a back-mutated and relaxed structure of the calcium-loaded recoverin mutant (PDB ID 1LA3) agreed well with the $\Delta G_{\mathrm{b}}^{\mathrm{o}}$ of $-9.5 \mathrm{kcal} / \mathrm{mol}$ derived from the experimentally measured microscopic dissociation constant of the EF3 loop in the open state of recoverin. ${ }^{12}$ To account for the diversity of conformations of the semi-open state without $\mathrm{Ca}^{2+}$, we considered two additional semi-open recoverin structures. These geometries were extracted from the REST2 trajectory of the semi-open state lacking $\mathrm{Ca}^{2+}$, the first (denoted as structure A) being structurally similar to the calcium-loaded recoverin mutant and the second (structure B) featuring differently rotated domains and a different relative angle of the two EF3 helices (see Fig. 2).

Recoverin- $\mathrm{Ca}^{2+}$ complexes that formed spontaneously in MD trajectories did not exhibit strong binding (see Table 1) as the $\mathrm{Ca}^{2+}$ coordination number did not exceed 4. However, when reshaping of the EF3 loop around $\mathrm{Ca}^{2+}$ was accelerated in a biased simulation, we observed strong calcium binding for the former of the two structures, with $\Delta G_{\mathrm{b}}^{\mathrm{o}}$ of equaling $-9.6 \mathrm{kcal} / \mathrm{mol}$. This binding energy was in good agreement with the value obtained for the calcium-loaded recoverin mutant, as well as with the experimental result. In contrast, the significantly different geometry of the two EF3 helices did not allow for achieving full $\mathrm{Ca}^{2+}$ coordination in the latter structure in the biased simulation.

To rationalize the differences in the calculated binding energies, we first remark that the $\mathrm{Ca}^{2+}$ ion is coordinated by five residues in the EF3 loop of the semi-open NMR structure, ${ }^{14}$ namely Asp 110, Asp 112, Asn 114, Thr 116, and Glu 121. The last of these residues interacted with $\mathrm{Ca}^{2+}$ in a bidentate fashion in our simulation (see Fig. 2), resulting in a $\mathrm{Ca}^{2+}$ coordination number of six in the 
free-energy minimum (Table 1). We also observed that, on average, one water molecule was in contact with $\mathrm{Ca}^{2+}$ in addition to the loop residues (Table 1). The six loop oxygen atoms together with that of the water molecule created a pentagonal bipyramid around the $\mathrm{Ca}^{2+}$ ion, a configuration characteristic of the $\mathrm{Ca}^{2+}$ binding to EF hands of calcium-sensing proteins. ${ }^{17}$ In this configuration, the EF3 loop was tightly packed around the calcium, and helix F (the entering helix of EF3) was close to perpendicular to helix $\mathrm{G}$ (the exiting helix of EF3), with the C-terminal end of the former being positioned close to the latter. In contrast, the C-terminal end of helix F was pulled away from helix $\mathrm{G}$ in the closed state, owing to the interaction of the former helix with helices E and A, located proximally to it in the closed structure. As a consequence, the full coordination of the $\mathrm{Ca}^{2+}$ ion by the loop residues became less energetically favorable than in the semi-open state, leading to a weaker $\mathrm{Ca}^{2+}$ binding and to a decrease in the average coordination number to 5.1 (see Table 1 and Fig. 2). Interestingly, this coordination number was slightly higher than 4.9 observed for the semi-open structure A from REST2, despite the fact that the $\mathrm{Ca}^{2+}$ binding to this structure was at least $3 \mathrm{kcal} / \mathrm{mol}$ stronger than to the closed state. A detailed examination reveals that the EF3 loop of structure A, while exhibiting a similar arrangement of the side chains as the semi-open NMR structure, became somewhat elongated, such that an extra water molecule could insert itself between $\mathrm{Ca}^{2+}$ and Glu 121 (see Fig. S15). Thus, Glu 121 did not coordinate $\mathrm{Ca}^{2+}$ directly, but via a bridge formed by an additional water molecule. The high stability of this arrangement resulted in a $\Delta G_{\mathrm{b}}^{\mathrm{o}}$ that did not differ substantially from that obtained for the semiopen NMR structure. Finally, the weak $\mathrm{Ca}^{2+}$ binding to the semi-open structure B from REST2 was due to the geometry of helix F, which became shorter and more distant from helix G than in the semiopen NMR structure, thereby producing an extended conformation of the EF3 loop, unfavorable to the binding of $\mathrm{Ca}^{2+}$.

Our results demonstrate that a conformational transition to the semi-open state gives rise to structures that can bind a $\mathrm{Ca}^{2+}$ ion in EF3 by $3-6 \mathrm{kcal} / \mathrm{mol}$ more strongly than in the closed state, which corresponds to a 200 to 20000 times lower dissociation constant. However, not all structures from the conformational ensemble of the semi-open state display the same high affinity for calcium. An increased concentration of $\mathrm{Ca}^{2+}$ ions favors structures with a more perpendicular relative orientation of the EF3 helices, i.e., such that allow the EF3 loop to fully coordinate the $\mathrm{Ca}^{2+}$ ion. 
For comparison, we also calculated the $\Delta G_{\mathrm{b}}^{\mathrm{o}}$ for the EF loop 2 in an open state of recoverin loaded with two $\mathrm{Ca}^{2+}$ ions (PDB ID 1JSA). ${ }^{9}$ In line with earlier experimental data, ${ }^{12}$ we confirmed that the binding to EF2 is weaker than to EF3 (Table 2). Accordingly, we observed that the average $\mathrm{Ca}^{2+}$ coordination by the oxygen atoms of the EF2 loop was 5, i.e., lower than in EF3.

\begin{tabular}{|c|c|c|c|c|}
\hline State & Structure & $\begin{array}{c}\Delta G_{\mathrm{b}}^{\mathbf{0}} \\
{[\mathrm{kcal} / \mathrm{mol}]}\end{array}$ & $\mathrm{CN}_{\text {loop }}$ & $\mathrm{CN}_{\text {water }}$ \\
\hline Closed & $\begin{array}{l}\text { NMR structure, } \\
\text { spontaneous binding }\end{array}$ & $-4.8 \pm 1.2$ & 3.6 & 3.2 \\
\hline Closed & $\begin{array}{l}\text { NMR structure, } \\
\text { after EF3 adaptation }\end{array}$ & $-6.3 \pm 1.9$ & 5.1 & 1.7 \\
\hline Semi-open & NMR structure & $-11.1 \pm 0.4$ & 6.0 & 1.0 \\
\hline Semi-open & $\begin{array}{l}\text { from REST2 without } \mathrm{Ca}^{2+} \text { (structure A), } \\
\text { spontaneous binding }\end{array}$ & $-3.4 \pm 0.4$ & 1.9 & 4.7 \\
\hline Semi-open & $\begin{array}{c}\text { from REST2 without } \mathrm{Ca}^{2+} \text { (structure A), } \\
\text { after EF3 adaptation }\end{array}$ & $-9.6 \pm 0.9$ & 4.9 & 2.0 \\
\hline Semi-open & $\begin{array}{l}\text { from REST2 without } \mathrm{Ca}^{2+} \text { (structure } \mathrm{B} \text { ), } \\
\text { spontaneous binding }\end{array}$ & $-5.1 \pm 0.4$ & 2.9 & 3.3 \\
\hline Open & $\begin{array}{l}\text { experiment on non-myristoylated } \\
\text { recoverin }\end{array}$ & -9.5 & $\mathrm{n} / \mathrm{a}$ & $\mathrm{n} / \mathrm{a}$ \\
\hline
\end{tabular}

Table 1. Standard $\mathrm{Ca}^{2+}$ binding free energies $\Delta G_{\mathrm{b}}^{\mathrm{o}}$ to $\mathrm{EF} 3$, average $\mathrm{Ca}^{2+}$ coordination numbers $\mathrm{CN}_{\text {loop }}$ due to loop residues, and average numbers of water molecules $\mathrm{CN}_{\text {water }}$ interacting with $\mathrm{Ca}^{2+}$ in the free-energy minimum.

\begin{tabular}{c|c|r|r|r} 
State & Structure & $\Delta \boldsymbol{G}_{\mathbf{b}}^{\mathbf{o}}[\mathbf{k c a l} / \mathbf{m o l}]$ & \multicolumn{1}{|c|}{$\mathbf{C N}_{\text {loop }}$} & \multicolumn{1}{|c}{$\mathbf{C N}_{\mathbf{w a t e r}}$} \\
\hline Open & NMR structure & $-6.2 \pm 0.3$ & 5.0 & 2.0 \\
\hline Open & $\begin{array}{c}\text { experiment on non-myristoylated } \\
\text { recoverin }\end{array}$ & -7.0 & $\mathrm{n} / \mathrm{a}$ & $\mathrm{n} / \mathrm{a}$
\end{tabular}

Table 2. Standard $\mathrm{Ca}^{2+}$ binding free energies $\Delta G_{\mathrm{b}}^{\mathrm{o}}$ to $\mathrm{EF} 2$, average $\mathrm{Ca}^{2+}$ coordination number $\mathrm{CN}_{\text {loop }}$ due to loop residues, and average number of water molecules $\mathrm{CN}_{\text {water }}$ interacting with $\mathrm{Ca}^{2+}$ in the free-energy minimum. 

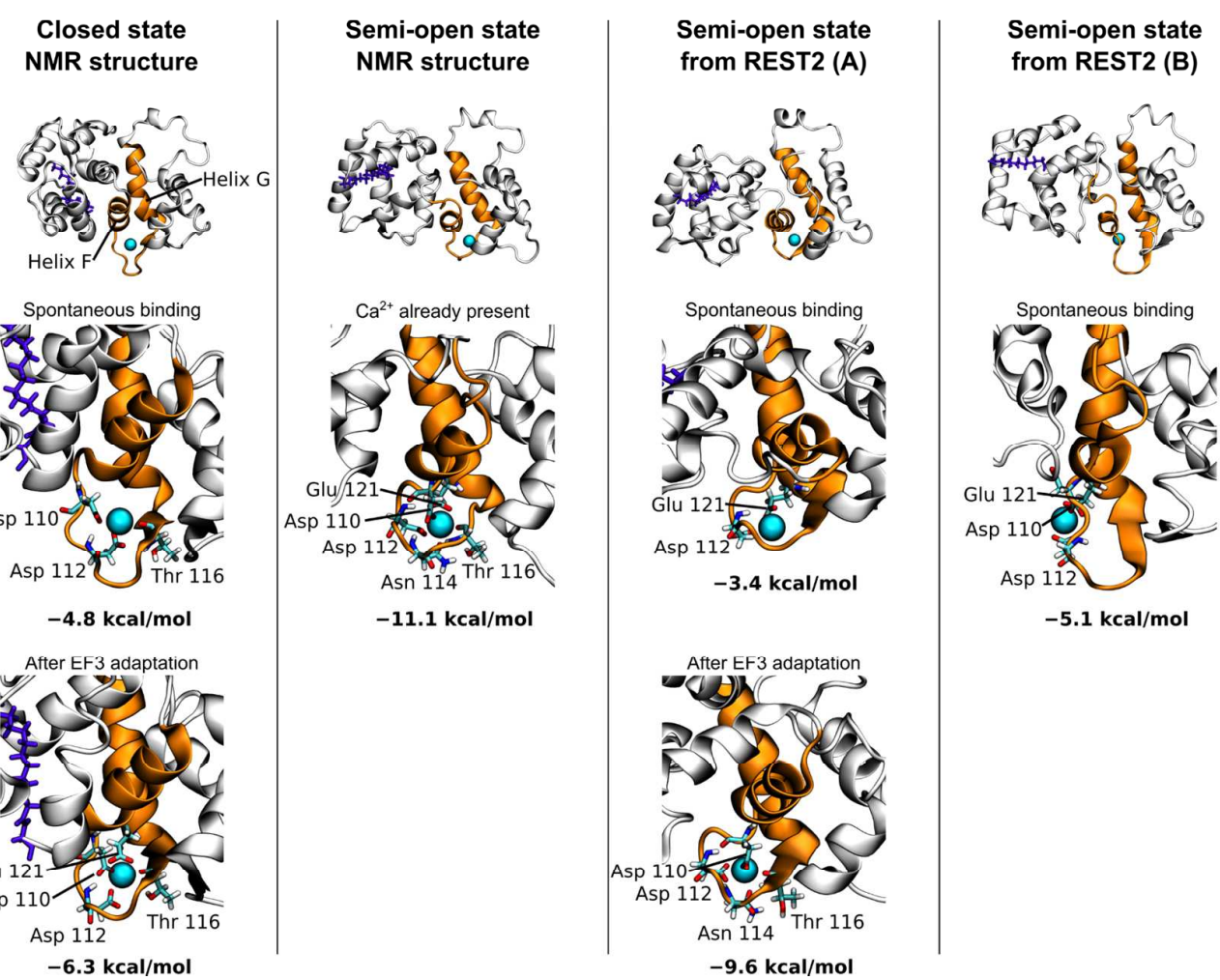

Figure 2. Different conformations of recoverin exhibiting varying $\mathrm{Ca}^{2+}$ binding affinities to EF3 (highlighted in orange). The detailed views of EF3 show amino-acid residues typically coordinating the $\mathrm{Ca}^{2+}$ ion in the free-energy minima.

The present results, in combination with the available NMR data and the results of $\mathrm{Ca}^{2+}$ binding assays, offer a detailed picture of the $\mathrm{Ca}^{2+}$-induced myristoyl switch, which operates as follows. Even at low $\mathrm{Ca}^{2+}$ concentrations, recoverin oscillates between its closed state and a semi-open state. The latter state, however, is not structurally uniform, but rather it exhibits a relatively broad range of domain orientations and conformations of the EF hands 3 and 2. Calcium binding to EF3 stabilizes a compact conformation of EF3, thereby making also the relative orientation of the two protein domains more stable and allowing the adjacent EF2 loop to adopt a position and conformation favorable to the next $\mathrm{Ca}^{2+}$ binding event. Binding of this second $\mathrm{Ca}^{2+}$ ion promotes a conformational change in the N-terminal domain which ultimately leads to an extrusion of the myristoyl moiety from inside the protein, accomplishing thus the biological function of recoverin. 
In this study, we shed light on the detailed mechanism of the function of recoverin as a myristoyl switch using advanced methods of molecular dynamics simulations in direct relation to NMR structural experiments and biochemical calcium binding essays. Our results underline the importance of the semi-open intermediate state as a crucial step of the $\mathrm{Ca}^{2+}$-induced myristoyl switch. While the semi-open state without $\mathrm{Ca}^{2+}$ is highly structurally variable leading to different calcium binding affinities to the EF3 loop, the most favorable structures bind $\mathrm{Ca}^{2+}$ by $3-6 \mathrm{kcal} / \mathrm{mol}$ more strongly than in the closed state. This results in a two- to four-orders of magnitude lower calcium dissociation constant in the semi-open state compared to the closed one. We can thus conclude that the myristoyl switch in recoverin works as follows: An increase in cytosolic calcium concentration stabilizes the semi-open state with strong calcium binding to the EF3 loop. The latter state is then able to bind a second calcium to the EF2 loop, leading to the open state with extruded myristoyl chain, which can be then incorporated from the cytosol into the rod outer-segment disk membrane.

\section{Methods}

Atomistic MD simulations were performed using the GROMACS 5.1.4 package. $^{18}$ The simulation box contained one recoverin molecule placed in a cubic box with length of $\sim 8.5 \mathrm{~nm}$, which was filled with a $150 \mathrm{mM} \mathrm{KCl}$ aqueous solution and which included additional $\mathrm{K}^{+}$ions to neutralize the whole system. A solution NMR structure of the E85Q mutant (PDB ID 1LA3, structure no. 1) ${ }^{14}$ after a back-mutation was used as a starting point for simulations of the semi-open state of recoverin. Simulations of the closed $\mathrm{Ca}^{2+}$-free state were started from a structure obtained from NMR measurements at zero $\mathrm{Ca}^{2+}$ concentration (PDB ID 1IKU, structure no. 1). ${ }^{7}$ Neither of these NMR structures contained the C-terminal segment (residues 190-202), as its conformation was not resolved in the NMR experiments. However, the absence of the C-terminal segment should not have any substantial impact on the mechanism of the conformational transition since the $\mathrm{Ca}^{2+}$-induced myristoyl switch was shown experimentally to be functional even for a C-terminally truncated recoverin construct. ${ }^{19}$ For simulations of the fully $\mathrm{Ca}^{2+}$-loaded open state, we used an equilibrated structure which we obtained previously ${ }^{11}$ from structure no. 8 of PDB ID 1JSA 9 . 
The protein was parameterized using the AMBER ff99SB-ILDN force field ${ }^{20}$, while water molecules were described with the TIP3P model. ${ }^{21}$ The force field parameters for the myristoyl moiety were the same as in our previous work. ${ }^{11}$ Force field parameters for calcium, chloride, and potassium ions were taken from refs. ${ }^{22-23}$ In line with the Electronic Continuum Correction (ECC) approach, ${ }^{24-25}$ the charges of $\mathrm{Ca}^{2+}$ as well as of all $\mathrm{K}^{+}$and $\mathrm{Cl}^{-}$ions were scaled by a factor of 0.75 to account for the electronic polarizability of the surrounding water molecules. In order to avoid altering the net charge of the calcium-loaded EF loops (EF2 and EF3), we evenly distributed the excess charge of $+0.5 e$ among 10 (or 8) oxygen atoms potentially coordinating the calcium ion in each loop. The binding of calcium to proteins was explored already in our previous paper, ${ }^{25}$ where we showed that the charge scaling procedure, accompanied by the redistribution of the positive charge among the loop atoms, leads to a significantly improved agreement with the experimental calcium binding free energies to the EF hands of calmodulin.

Allosteric effects of the EF3 loop conformation were explored using the REST2 technique ${ }^{16}$ by elevating the effective temperature of the loop residues to up to $900 \mathrm{~K}$. Free energy profiles of $\mathrm{Ca}^{2+}$ binding to EF3 and EF2 were obtained by employing the REUS technique. ${ }^{15}$ Both our REST2 and REUS simulations were performed in GROMACS 5.1.4 ${ }^{18}$ patched with the PLUMED 2.3.0 package. ${ }^{26}$ The VMD program ${ }^{27}$ was used to visualize the system and to prepare figures. Additional details on the simulation setup, force field parameters, system building, and structure equilibration can be found in SI.

\section{Acknowledgments}

We thank to Rieko Ishima for providing the recoverin NMR relaxation data. P.J. acknowledges support from the Czech Science Foundation (grant no. 16-01074S). This work was supported by The Ministry of Education, Youth and Sports from the Large Infrastructures for Research, Experimental Development and Innovations project „IT4Innovations National Supercomputing Center - LM2015070“. Access to computing and storage facilities owned by parties and projects contributing to the National Grid Infrastructure MetaCentrum provided under the program "Projects of Large Research, Development, and Innovations Infrastructures" (CESNET 
LM2015042), is greatly appreciated. P.S was supported by MEYS CR project LO1304. O.H.S.O. acknowledges financial support from Integrated Structural Biology Research Infrastructure of Helsinki Institute of Life Science (Instruct-HiLIFE).

\section{Associated Content \\ Supporting Information}

Further details on computational methods and additional analysis of MD trajectories.

\section{References}

1. Clapham, D. E. Calcium Signaling. Cell 2007, 131, 1047-1058.

2. Burgoyne, R. D.; Callaghan, D. W. O.; Hasdemir, B.; Haynes, L. P.; Tepikin, A. V. Neuronal $\mathrm{Ca}^{2+}$-Sensor Proteins: Multitalented Regulators of Neuronal Function. Trends Neurosci. 2004, 27, 203-209.

3. Burgoyne, R. D. Neuronal Calcium Sensor Proteins: Generating Diversity in Neuronal $\mathrm{Ca}^{2+}$ Signalling. Nat. Rev. Neurosci. 2007, 8, 182-193.

4. Ames, J. B.; Lim, S. Molecular Structure and Target Recognition of Neuronal Calcium Sensor Proteins. Biochim. Biophys. Acta 2012, 1820, 1205-1213.

5. Ames, J. B.; Levay, K.; Wingard, J. N.; Lusin, J. D.; Slepak, V. Z. Structural Basis for Calcium-Induced Inhibition of Rhodopsin Kinase by Recoverin. J. Biol. Chem. 2006, 281, 37237-37245.

6. Chazin, W. J. Relating Form and Function of EF-Hand Calcium Binding Proteins. Acc. Chem. Res. 2011, 44, 171-179.

7. Tanaka, T.; Ames, J. B.; Harvey, T. S.; Stryer, L.; Ikura, M. Sequestration of the Membrane-Targeting Myristoyl Group of Recoverin in the Calcium-Free State. Nature 1995, 376, 444-447.

8. Ames, J. B.; Tanaka, T.; Ikura, M.; Stryer, L. Nuclear Magnetic Resonance Evidence for $\mathrm{Ca}^{2+}$-Induced Extrusion of the Myristoyl Group of Recoverin. J. Biol. Chem. 1995, 270, 3090930913.

9. Ames, J. B.; Ishima, R.; Tanaka, T. Molecular Mechanics of Calcium-Myristoyl Switches. Nature 1997, 389, 198-202.

10. Valentine, K. G.; Mesleh, M. F.; Opella, S. J.; Ikura, M.; Ames, J. B. Structure, Topology, and Dynamics of Myristoylated Recoverin Bound to Phospholipid Bilayers. Biochemistry 2003, 42, 6333-6340.

11. Timr, S.; Pleskot, R.; Kadlec, J.; Kohagen, M.; Magarkar, A.; Jungwirth, P. Membrane Binding of Recoverin: From Mechanistic Understanding to Biological Functionality. ACS Cent. Sci. 2017, 3, 868-874.

12. Ames, J. B.; Porumb, T.; Tanaka, T.; Ikura, M.; Stryer, L. Amino-Terminal Myristoylation Induces Cooperative Calcium-Binding to Recoverin. J. Biol. Chem. 1995, 270, 4526-4533. 
13. Xu, X.; Ishima, R.; Ames, J. B. Conformational Dynamics of Recoverin's Ca ${ }^{2+}$-Myristoyl Switch Probed by ${ }^{15} \mathrm{~N}$ NMR Relaxation Dispersion and Chemical Shift Analysis. Proteins 2011, 79, 1910-1922.

14. Ames, J. B.; Hamasaki, N.; Molchanova, T. Structure and Calcium-Binding Studies of a Recoverin Mutant (E85Q) in an Allosteric Intermediate State. Biochemistry 2002, 41, 57765787.

15. Sugita, Y.; Akio, K.; Yuko, O. Multidimensional Replica-Exchange Method for FreeEnergy Calculations. J. Chem. Phys. 2000, 113, 6042-6051.

16. Wang, L.; Friesner, R. A.; Berne, B. J. Replica Exchange with Solute Scaling: A More Efficient Version of Replica Exchange with Solute Tempering (REST2). J. Phys. Chem. B 2011, 115, 9431-9438.

17. Strynadka, N. C. J.; James, M. N. G. Crystal Structures of the Helix-Loop-Helix CalciumBinding Proteins. Annu. Rev. Biochem 1989, 58, 951-999.

18. Abraham, M. J.; Murtola, T.; Schulz, R.; Páll, S.; Smith, J. C.; Hess, B.; Lindahl, E. Gromacs: High Performance Molecular Simulations through Multi-Level Parallelism from Laptops to Supercomputers. SoftwareX 2015, 1-2, 19-25.

19. Weiergraber, O. H.; Senin, I. I.; Zernii, E. Y.; Churumova, V. A.; Kovaleva, N. A.; Nazipova, A. A.; Permyakov, S. E.; Permyakov, E. A.; Philippov, P. P.; Granzin, J.; Koch, K. W. Tuning of a Neuronal Calcium Sensor. J. Biol. Chem. 2006, 281, 37594-37602.

20. Lindorff-Larsen, K.; Piana, S.; Palmo, K.; Maragakis, P.; Klepeis, J. L.; Dror, R. O.; Shaw, D. E. Improved Side-Chain Torsion Potentials for the Amber ff99SB Protein Force Field. Proteins: Struct. Funct. Bioinform. 2010, 78, 1950-1958.

21. Jorgensen, W. L.; Chandrasekhar, J.; Madura, J. D.; Impey, R. W.; Klein, M. L. Comparison of Simple Potential Functions for Simulating Liquid Water. J. Chem. Phys. 1983, 79, 926-935.

22. Kohagen, M.; Mason, P. E.; Jungwirth, P. Accurate Description of Calcium Solvation in Concentrated Aqueous Solutions. J. Phys. Chem. B 2014, 118, 7902-7909.

23. Chang, T. M.; Dang, L. X. Detailed Study of Potassium Solvation Using Molecular Dynamics Techniques. J. Phys. Chem. B 1999, 103, 4714-4720.

24. Leontyev, I.; Stuchebrukhov, A. Accounting for Electronic Polarization in NonPolarizable Force Fields. Phys. Chem. Chem. Phys. 2011, 13, 2613-2626.

25. Kohagen, M.; Lepsik, M.; Jungwirth, P. Calcium Binding to Calmodulin by Molecular Dynamics with Effective Polarization. J. Phys. Chem. Lett. 2014, 5, 3964-3969.

26. Tribello, G. A.; Bonomi, M.; Branduardi, D.; Camilloni, C.; Bussi, G. PLUMED 2: New Feathers for an Old Bird. Comput. Phys. Commun. 2014, 185, 604-613.

27. Humphrey, W.; Dalke, A.; Schulten, K. VMD: Visual Molecular Dynamics. J. Mol. Graphics Modell. 1996, 14, 33-38. 